\title{
Microarray Analysis of microRNAs Expression Profiles in Adult and Aged Mice Hippocampus
}

\author{
Jia Yang1, Yuan Liu², Ling Wang², Zhenyin Yu², Cheng Wang², Yun Zheng3, Yang Liu²* \\ ${ }^{1}$ The Second Clinical Medical College of Yangtze University/The Department of Radiology, Jingzhou Central Hospital, Jingzhou, \\ China \\ ${ }^{2}$ Department of Pathogenic Biology, School of Medicine, Yangtze University, Jingzhou, China \\ ${ }^{3}$ Department of Medical Function, School of Medicine, Yangtze University, Jingzhou, China \\ Email: *weijiaershe@163.com, *oceanerliu@aliyun.com
}

How to cite this paper: Yang, J., Liu, Y., Wang, L., Yu, Z.Y., Wang, C., Zheng, Y. and Liu, Y. (2017) Microarray Analysis of microRNAs Expression Profiles in Adult and Aged Mice Hippocampus. Yangtze Medicine, 1, 216-234.

https://doi.org/10.4236/ym.2017.14022

Received: September 19, 2017

Accepted: December 10, 2017

Published: December 13, 2017

Copyright $\odot 2017$ by authors and Scientific Research Publishing Inc. This work is licensed under the Creative Commons Attribution International License (CC BY 4.0).

http://creativecommons.org/licenses/by/4.0/ Open Access

\begin{abstract}
Purpose: To analyze the miRNA expression profiles in C57 mice ageing hippocampus in detail, and investigate the functional information of these hippocampus specific miRNAs and the related regulatory networks. Methods: Microarrays were used to analyze miRNA expression profiles in adult and aged hippocampi, and bioinformatics analysis methods, such as three public datasets (Mirbase, Miranda, and Mirdb), DAVID online tools and KEGG pathway tools were used to study in detail the target genes of the differentially expressed miRNAs. Results: 26 differentially expressed miRNAs were identified by greater than 1.5 -fold change (intersection of two sets), of which 16 were up-regulated and 10 were down-regulated. DAVID Functional Annotation Cluster (FAC) analysis of the 132 predicted target genes of up-regulated miRNAs revealed confident enrichment scores for synaptic function and apoptosis etc. (Figure 1), indicating the functional significance and importance of these miRNAs during hippocampal ageing. Conclusions: Bioinformatic analyses of the differentially expressed miRNAs have identified a number of miRNAs with putative involvement in the hippocampus ageing process. This study lays a solid foundation for further studies to clarify the important regulation function of miRNAs in ageing process of brain tissue.
\end{abstract}

\section{Keywords}

microRNA, Microarray, Hippocampus, Ageing

\section{Introduction}

Neurodegenerative disease is one of the leading causes of death in the worldwide, including Alzheimer's disease (AD), Parkinson's disease (PD) etc. They are 
the progressive development of deadly complex diseases, and many factors such as heredity and environment participate in these diseases process [1]. Epidemiological and biomedical studies have demonstrated that both genetic and age-related factors are crucial for the development and progression of these disorders. Attempts to understand the underlying mechanism of functional alterations in neural circuits during "normal aging" are receiving considerable attention and should provide new insights toward preventing and treating age-related disorders. However, our knowledge about whether and how neural circuits are remodeled and/or maintained during normal aging is still very limited [2].

The microRNAs (miRNAs) are a group of small noncoding RNAs that are single stranded chains consisting of 19 - 25 nucleotides ( 22 nucleotides) and transcribed by RNA polymerase II or III in the nucleus [3]. More and more studies showed that miRNAs play an important role in gene regulations by binding to the 3'-untranslated regions (3'-UTRs) of target messenger RNAs (mRNAs), resulting in post-transcriptional silencing by translational repression, mRNA degradation, or a combination of the two [4] [5] [6].

Emerging evidence indicates that miRNAs are actively involved in regulating gene expression patterns in the adult and aging brain [7] [8] [9]. Ageing remains the most robust risk factor, thus far identified for human neurodegenerative conditions. Mechanisms of ageing under the regulation of miRNAs may contribute to neurodegenerative diseases pathology, and systemic increase in specific miRNAs may suppress various cellular functions in neurodegenerative diseases, such as redox defenses or DNA repair mechanisms, by targeting mRNA and/or protein species in brain and peripheral tissues [10].

miRNA microarray technology is an efficient method to generate miRNA expression profiles, and these data can be used to extract information regarding the regulatory pathways initiated by miRNAs, especially in the case of regulation due to degradation, by integrating the mRNA expression profiles of the predicted miRNA target genes. Such an approach has been applied to study the functional linkage between miRNAs and physiological or pathological processes [11] [12] [13]. Recent estimates put the number of human miRNAs at 1100 or more, composing complex regulatory networks that influence the expression of as many as two thirds of all genes [14]. Such a large family of genes could explain some of the difficulties that neurobiologists generally have encountered in their efforts to link individual miRNA genes to certain mental disorders. Because they are single molecular entities that dictate the expression of fundamental regulatory pathways, miRNAs represent potential drug targets of unprecedented power.

Neurodegenerative diseases are a type of nervous system disorders in which neurons are lost progressively with age, resulting in the loss of multiple functions such as behavior and memory, among others [15]. The hippocampus is an important part of the limbic system that plays vital role in the behavioral, emotional and memory processes [16]. C57 mouse is a general animal model for biological research to investigate the miRNAs expression profiles in C57 mouse 
hippocampus in detail, and we deliberately selected adult and aged C57 mice hippocampus to analyze the miRNA expression profiles.

Furthermore, we investigated the functional information of these hippocampus specific miRNAs and the related regulatory networks, studied in detail the target genes of the selected miRNAs predicted by three public datasets (Mirbase, Miranda, and Mirdb) through function and pathway enrichment analysis, and thus developed a global view of C57 mouse ageing hippocampus specific miRNA expression profiles and their target maps.

\section{Materials and Methods}

\subsection{Animals}

Adult male (10 months old and 20 months old) C57 mice were obtained from Hubei provincial center for disease control and prevention (Wuhan, China). The animals were acclimated for 5-7 days under standard conditions and kept for appropriate age. Ten 10 months old and Ten 20 months old C57 mice were killed, and the brains were quickly removed. Three of them were used for chip assay, and the remaining seven were used for quantitative real time PCR (qRTPCR) verification in each group. Fresh hippocampi were then dissected from the brain on a chilled glass plate on ice according to the procedures described in reference [17]. The hippocampi were stored at $70^{\circ} \mathrm{C}$ until the day of assay. All animal experiments in this study were approved by Medical Ethics Committee of Yangtze University and carried out in accordance with the guiding principles for the care and use of laboratory animals published by the U.S. National Institutes of Health (NIH Publication No.85-23, revised 1996) and the ARRIVE guidelines.

miRNA microarray

Microarray analysis was performed by Kangcheng Bio-tech Inc. (Shanghai, China). Briefly, total RNA was harvested using Trizol (Invitrogen, Carlsbad, CA, USA) and miRNeasy mini kit (Qiagen, Valencia, CA, USA) according to manufacturer's instructions. After RNA quantity measurement with the NanoDrop 1000 , the samples were labeled using the miRCURYTM Hy3TM/Hy5 ${ }^{\mathrm{TM}}$ Power labeling kit and hybridized on the miRCURYTM LNA Array (v.16.0, Exiqon, Skelstedet, Vedbaek, Denmark). After hybridization, scanning was performed with the Axon GenePix 4000B microarray scanner (Molecular Devices, Downingtown, PA, USA). The raw intensity of the image was read with GenePix pro V6.0 (Molecular Devices) and the intensity of green signal was calculated after background subtraction. Four replicated spots of each probe on the same slide were averaged. Median Normalization Method was used to obtain "Normalized Data": Normalized Data $=($ Foreground - Background $) /$ median, where the median was the $50 \%$ quantile of miRNA intensity, which was larger than 50 in all samples after background correction.

\subsection{Quantitative RT-PCR Validation}

Total RNA was extracted using Trizol reagent (Invitrogen). cDNA was synthe- 
sized using the PrimeScript RT Reagent Kit (GeneCopoeia, MD, USA). A total of 6 differentially expressed miRNAs were selected based on their function and involvement in pathways and processes important to C57 mouse ageing. Detection of the mature form of miRNAs was performed using Quantitect SYBR Green PCR Kit (GeneCopoeia) and qRT-PCR Primer Sets (GeneCopoeia) with the U6 small nuclear RNA as an internal control.

\subsection{Bioinformatics Analysis of Normalized Microarray Data}

Up and down-regulated miRNAs of greater than 1.5 fold change from the 20month-old and 10-month-old were selected from miRNAs expression profiling data (Intersection of three sets of chip results).

Three types of miRNA target prediction software, i.e., Microcosm (http://www.ebi.ac.uk/enright-srv/microcosm/htdocs/targets/v5/), Miranda (http://www.microrna.org/microrna/home.do), Mirdb (http://mirdb.org/miRDB/) were used to predict the target genes of the selected miRNAs. The intersection of these three datasets was used as the prediction results of the target genes of the selected miRNAs.

\subsection{Clustering and Principal Component Analysis}

The hierarchical clustering method [18] was used to classify different group patterns. Principal component analysis (PCA) [19] was used to produce a two-dimensional graph of the distances between different groups.

Gene Ontology (GO) classification systems was used to assign putative function to each clone by way of biological process, molecular function and cellular components. The Database for Annotation, Visualization and Integrated Discovery (DAVID) v6.7b [20] was used to determine pathways and processes of major biological significance and importance through the Functional Annotation Cluster (FAC) tool based on the GO annotation function.

\subsection{DAVID Functional Annotation Cluster Analysis}

DAVID FAC analysis was conducted on two independent normalized gene lists containing the target genes (Intersection of 5 or more miRNAs) of 1.5-fold up-regulated normalized miRNAs and 1.5-fold down-regulated normalized miRNAs (5 or more). High stringency ease score parameters were selected, to indicate confident enrichment scores of functional significance and importance of the given pathways and processes investigated. The Gene Ontology (GO) system in DAVID was utilized to identify enriched biological themes in both gene lists.

\subsection{Mapping and Visual Pathway Analysis}

Kyoto Encyclopedia of Genes and Genomes (KEGG) pathway tools were used to visually map cluster of the target genes involved in common pathways and processes for both pathway-specific and molecular overview purposes. KEGG 
pathway tools were utilized through DAVID online tools. Since visual mapping was the primary objective, all target genes (Intersection of 5 or more miRNAs) of normalized miRNAs that were differentially expressed by 1.5 -fold were considered for the KEGG pathway analysis. Heat map analyses were also conducted through DAVID to produce a matrix of enriched GO terms with common target genes (Intersection of 5 or more miRNAs) of normalized miRNAs that were 1.5-fold or more up and down-regulated. The green and black shading on the heat map matrix indicates a positive and negative correlation between the enriched GO term and the given target gene, respectively.

\section{Result}

\subsection{All Differentially Expressed miRNAs}

The $6^{\text {th }}$ generation of miRNA array (Exiqon) contains about 1280 capture probes, covering mouse microRNAs annotated in miRBase 16.0. C57 mice hippocampi of 10 month old and 20 month old were used for miRNAs microarray analysis. Up and down-regulated miRNAs with change more than 1.5 fold from both 20-month-old and 10-month-old (Table 1, Table 2) were chosen for further study.

In total, two replicate microarray hybridizations were performed, and 26 differentially expressed miRNAs ( $>1.5$ fold) were identified through analysis. Of these miRNAs, 16 were up-regulated and 10 down-regulated during hippocampus ageing. The intersections of the predicted target genes from 5 or more selected miRNAs' were analyzed using the Functional Annotation Cluster (FAC) tool contained in the Database for Annotation, Visualization and Integrated Discovery (DAVID) [20]. DAVID FAC analysis of the 132 predicted target genes of up-regulated miRNAs ( $>1.5$-fold) produced a total of 31 enriched functional clusters under high stringency conditions (Enrichment Score $>1.5$ ). The enrichment score gives an indication of the biological significance of the gene groups being analyzed, and the top 10 considered in our study are cell fraction, synaptic function, regulation of secretion, apoptosis, response to hormone stimulus, protein localization, neuron projection, synaptic transmission, regulation of kinase activity and regulation of apoptosis (Figure 1). DAVID FAC analysis of the 268 predicted target genes of down-regulated miRNAs (>1.5-fold) produced a total of 44 enriched functional clusters under high stringency conditions (Enrichment Score > 1.5). The top 10 were shown in Figure 2. Some significant biological processes were same with the target genes of up-regulated miRNAs, maybe just be regulated and regulation in the opposite direction. Considering the negative regulation is the main characteristic of miRNA function, target genes of up-regulated miRNAs (>1.5-fold) were chosen for further analysis.

\subsection{Validation of Microarray Data by qRT-PCR}

Two microarray sets may not be enough to convince differential expression in adult and aged hippocampi, so qRT-PCR was used to validate microarray results. 
A total of 6 differentially expressed miRNAs were selected based on their function and involvement in pathways and processes important to C57 mice ageing. The qRT-PCR results are correlated with the microarray expression data (Figure 3, Table 1, Table 2).

\subsection{Synaptic Function}

Synaptic function activity showed a very high enrichment score in the FAC analysis of target genes of up-regulated miRNAs in 20 month old C57 mouse

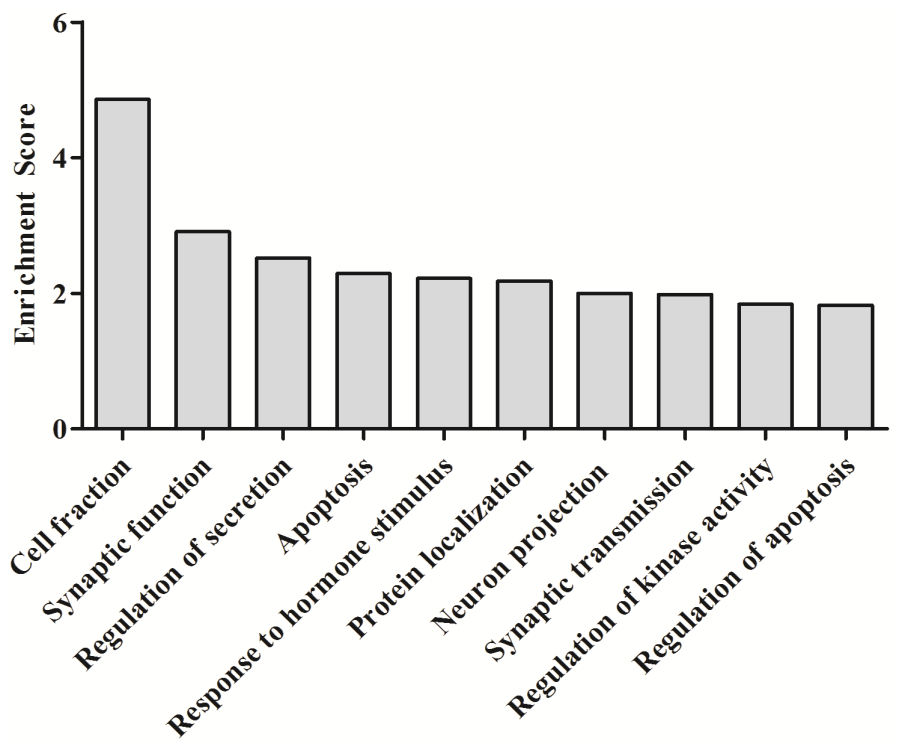

Figure 1. DAVID FAC analysis of target genes of 20 months old VS 10 months old up-regulated miRNAs ( $>1.5$ fold). Major FACs for up-regulated miRNAs. Significance is determined by corresponding enrichment scores.

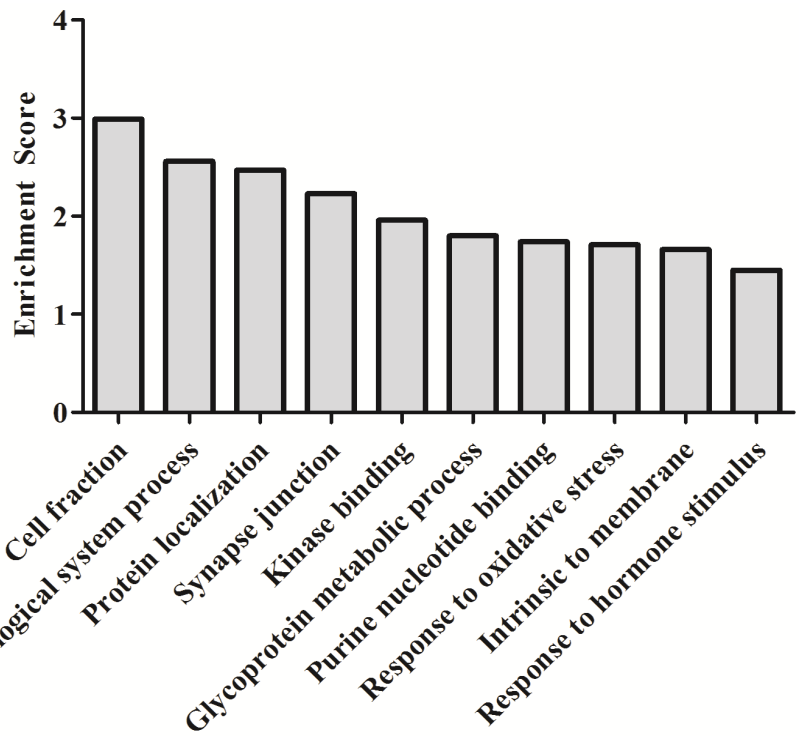

Figure 2. DAVID FAC analysis of target genes of 20 months old VS 10 months old downregulated miRNAs ( $>1.5$ fold). Major FACs for down-regulated miRNAs. Significance is determined by corresponding enrichment scores. 


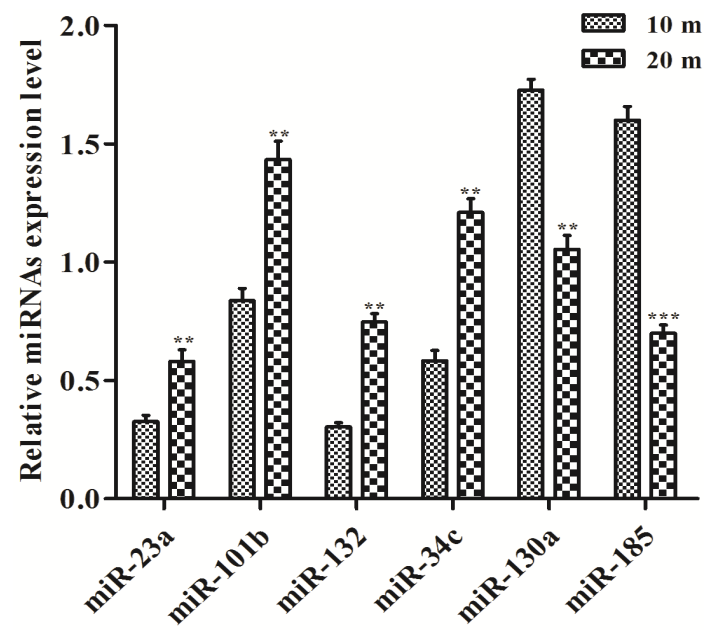

Figure 3. Validation of microarray data. A total of six miRNAs in our microarray experiments were selected and their relative expression determined using qRT-PCR. The bars represent relative levels (mean $\pm \mathrm{SEM}, \mathrm{n}=5$ ) of miRNAs normalized by U6 small nuclear RNA. $\left({ }^{\star *} \mathrm{P}<0.01,{ }^{* *} \mathrm{P}<0.001\right) .(10 \mathrm{~m}: 10$ months old; $20 \mathrm{~m}: 20$ months old).

Table 1. The miRNAs of 1.5 fold and above up-regulated in hippoccampus of 20 months old VS 10 months old mice (intersection of $20 \mathrm{~m}_{1} \mathrm{VS} 10 \mathrm{~m}_{1}$ and $20 \mathrm{~m}_{2} \mathrm{VS} 10 \mathrm{~m}_{2}$ ).

\begin{tabular}{ccc}
\hline Name & $20 \mathrm{~m}_{1}$ VS $10 \mathrm{~m}_{1}$ & $20 \mathrm{~m}_{2}$ VS $10 \mathrm{~m}_{2}$ \\
\hline mmu-miR-132 & 1.7613 & 2.4891 \\
mmu-miR-137* & 1.9709 & 1.6602 \\
mmu-miR-21* & 2.7865 & 1.6681 \\
mmu-miR-23a & 1.7312 & 1.8917 \\
mmu-miR-292-3p & 2.4510 & 2.2129 \\
mmu-miR-296* & 2.2620 & 1.6505 \\
mmu-miR-29b-1* & 1.6041 & 1.7299 \\
mmu-miR-345-3p & 1.5817 & 3.7024 \\
mmu-miR-101b & 1.7229 & 2.9498 \\
mmu-miR-106b & 3.0489 & 2.7027 \\
mmu-miR-125a-5p & 1.5474 & 2.2727 \\
mmu-miR-125b-5p & 2.6746 & 2.2726 \\
mmu-miR-15b & 1.7429 & 4.1893 \\
mmu-miR-195 & 3.2623 & 1.7513 \\
mmu-miR-23b & 2.2299 & 1.5384 \\
mmu-miR-34c & 3.1739 & 2.1052 \\
\hline
\end{tabular}

(10 m: 10 months old; $20 \mathrm{~m}: 20$ months old).

(Figure 1). DAVID heat map analyses identified 16 genes that functionally clustered into common GO terms related to synaptic transmission, synaptic plasticity, behavior, learning and memory, cognition and cell fraction (Figure 4(A)). A number of genes that were identified in the heat map, such as N-methyl-D-aspartate 
Table 2. The miRNAs of 1.5 fold and above down-regulated in hippoccampus of 20 months old VS 10 months old mice (intersection of $20 \mathrm{~m}_{1}$ VS $10 \mathrm{~m}_{1}$ and $20 \mathrm{~m}_{2}$ VS 10 $\left.\mathrm{m}_{2}\right)$.

\begin{tabular}{ccc}
\hline Name & $20 \mathrm{~m}_{1} \mathrm{VS} 10 \mathrm{~m}_{1}$ & $20 \mathrm{~m}_{2} \mathrm{VS} 10 \mathrm{~m}_{2}$ \\
\hline mmu-let-7b & 0.5769 & 0.5971 \\
mmu-let-7i & 0.4685 & 0.5020 \\
mmu-miR-128 & 0.3656 & 0.5766 \\
mmu-miR-130a & 0.5650 & 0.6307 \\
mmu-miR-138 & 0.6151 & 0.6021 \\
mmu-miR-185 & 0.3547 & 0.4373 \\
mmu-miR-25 & 0.6442 & 0.3536 \\
mmu-miR-30c & 0.6637 & 0.4582 \\
mmu-miR-423* & 0.3514 & 0.4470 \\
mmu-miR-9 & 0.2471 & 0.2480 \\
\hline
\end{tabular}

(10 m: 10 months old; $20 \mathrm{~m}: 20$ months old).

subtype of glutamate receptors (NMDA-R), brain derived neurotrophic factor (BDNF), dopamine receptor D1A, early growth response 1, solute carrier family 24 , and tachykinin receptor 1 , were found highly related to learning or memory, cognition, behavior, regulation of transmission of nerve impulse, synaptic transmission, regulation of neuronal synaptic plasticity, regulation of neurological system process and membrane fraction. Especially, N-methyl-D-aspartate subtype of glutamate receptors (NMDA-R) is known to play a major role in the induction of synaptic plasticity as well as in the acquisition of memory traces [21]. Impaired long-term potentiation (LTP) expression at hippocampal synapses of the aging brain has recently been linked to weaker NMDA-R activation [22]. And the neurotrophin BDNF has been shown to modulate the development and function of synapses in the nervous system [23]. BDNF is important in modulating dentate gyrus neurogenesis [24] and in synaptogenesis [25].

These results suggest differentially expressed miRNAs were involved in the regulation of hippocampus synaptic function by regulating the expression of their target genes.

\subsection{Cell Fraction and Regulation of Secretion}

Cell fraction showed the highest enrichment score in the FAC analysis with the target genes of up-regulated miRNAs (>1.5-fold) during the ageing (Figure 1). DAVID two dimensional figure analysis shows that 42 genes are correlated with cell fraction, membrane fraction, etc. of GO classifications (Figure 4(B)). Dopamine receptor D1A, metabotropic and ionotropic glutamate receptors (GluRs), tumour necrosis factor receptor (TNFR) etc. appeared in these genes. Regulation of secretion is another important function cluster (Figure 1), and 22 genes that functionally clustered into common GO terms pertinent to regulation 
A

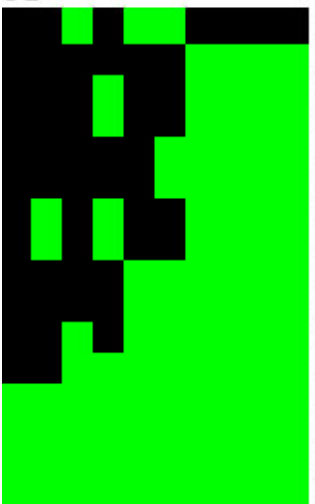

caspase 3 , apoptosis related cysteine protease

guanine nucleotide binding protein ( $G$ protein), alpha inhibiting 1

leucine zipper, putative tumor suppressor 1

tyrosine 3-monooxygenase/tryptophan 5-monooxygenase activation protein

sodium channel, voltage-gated, type $I X$, alpha

neurotrophic tyrosine kinase, receptor, type 2

candidate plasticity gene 1

steroidogenic acute regulatory protein

solute carrier family 1 (glial high affinity glutamate transporter), member 3

glutamate receptor, metabotropic 1

tachykinin receptor 1

solute carrier family 24 (sodiumipotassiumicalcium exchanger), member 2

dopamine receptor D1A

glutamate receptor, ionotropic, $N$-methyl D-aspartate $2 A$

brain derived neurotrophic factor

early growth response 1

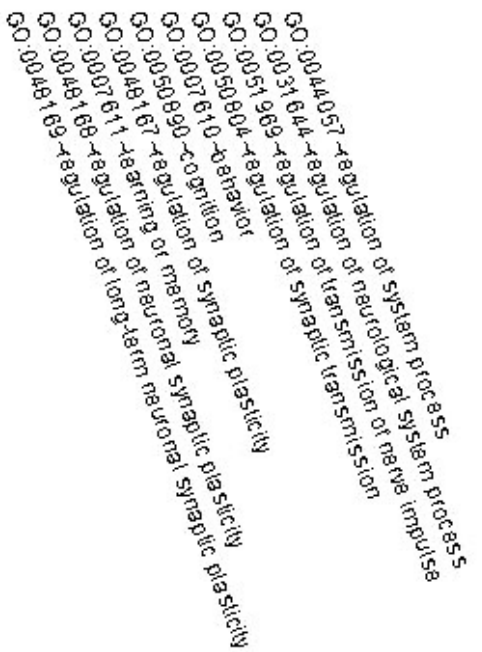

B

caspase 3 , apoptosis related cysteine protease

pyruvate kinase, liver and RBC

LIM domain kinase 2

similar to Annexin A? (Annexin VII) (Synexin); annexin A?

protein tyrosine phosphatase, non-receptor type 1

lecithin-retinol acyltransferase (phosphatidylcholine-retinol-0-acyltransferase)

somatostatin receptor 1

fragile $\times$ mental retardation 1

protein phosphatase 1 , regulatory (inhibitor) subunit 30

breast carcinoma amplified sequence 1

cytochrome $P 450$, family 7 , subfamily a, polypeptide 1

caspase 7

myotubularin related protein 3

regulator of G-protein signaling 4

dopamine receptor D1A

guanine nucleotide binding protein ( $G$ protein), alpha inhibiting 1 .

nephrosis 1 homolog, nephrin (human)

tumor necrosis factor receptor superfamily, member $1 \mathrm{~b}$

calciumicalmodulin-dependent serine protein kinase (MAGUK family)

protein phosphatase 1, regulatory (inhibitor) subunit $3 \mathrm{~B}$

glutamate receptor, ionotropic, $\mathrm{N}$-methyl $\mathrm{D}$-aspartate $2 \mathrm{~A}$

tumor protein $\mathrm{p} 73$-like

ATP-binding cassette, sub-family C (CFTR/MRP), member 5

carnitine palmitoyltransferase 1 a, liver

isoprenylcysteine carboxyl methyltransferase

adipocyte-specific adhesion molecule

adenylate cyclase 6

solute carrier family 1 (glial high affinity glutamate transporter), member 3

synaptosomal-associated protein 29

UDP glucuronosyltransferase 2 family, polypeptide B17

similar to stearoyl-coenzyme A desaturase 3 ; stearoyl-Coenzyme A desaturase 1

GDP dissociation inhibitor 1

sodium channel, voltage-gated, type II, alpha 1

$\mathrm{SH} 3$-domain kinase binding protein 1

vesicle-associated membrane protein 3

seven in absentia 2

solute carrier family 12 (sodiumipotassiumichloride transporters), member 2

solute carrier family 24 (sodiumipotassiumicalcium exchanger), member 2

glutamate receptor, metabotropic 1

similar to Septin-2 (Protein NEDD5)

dynamin 1-like

syntaxin 1B

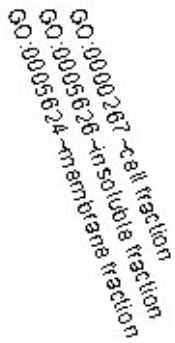


C

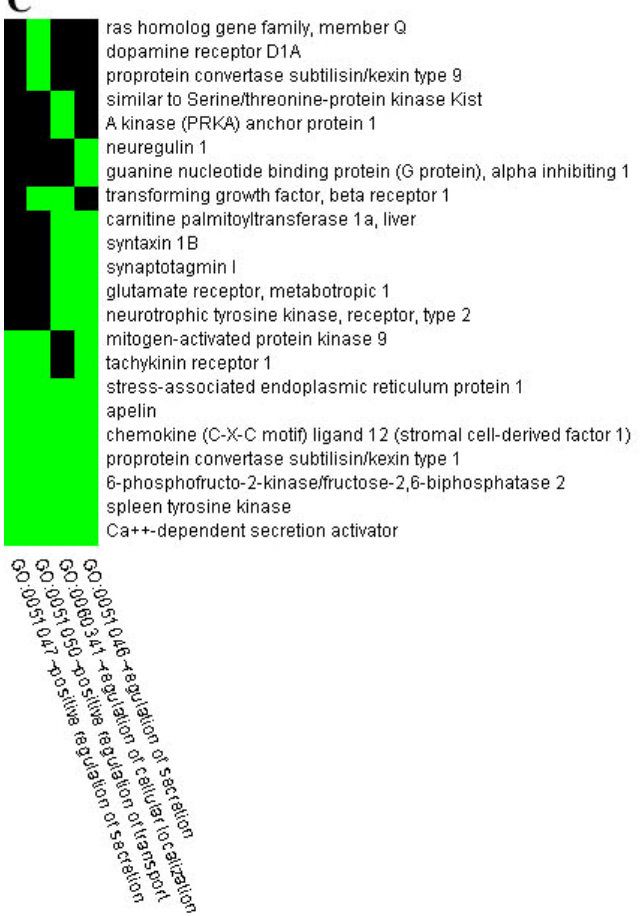

$\mathbf{E}$

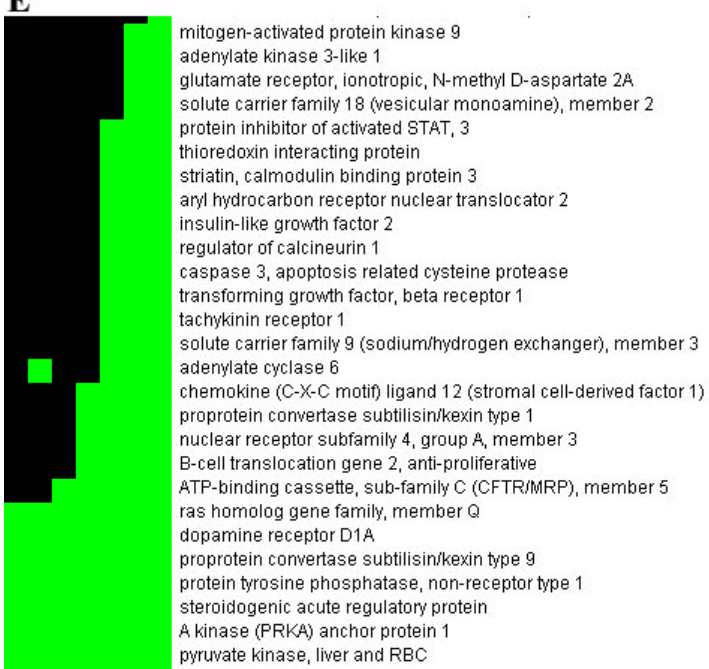

8888888

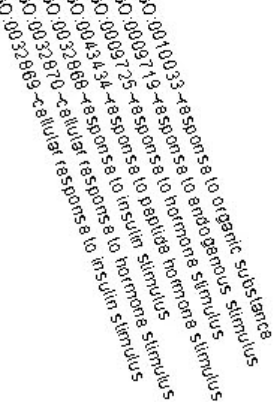

D

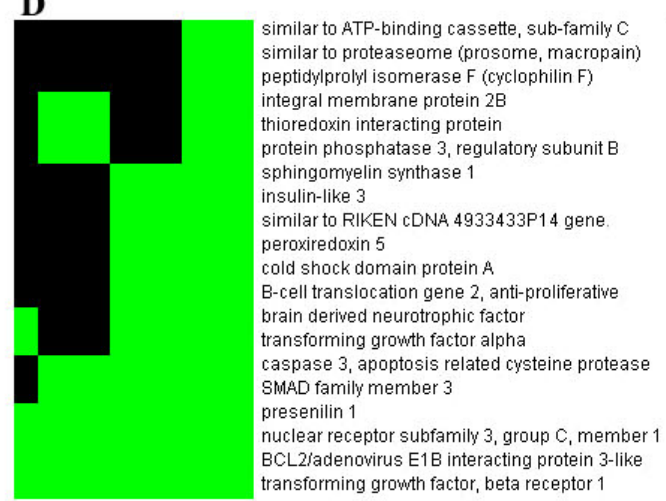

8888888888

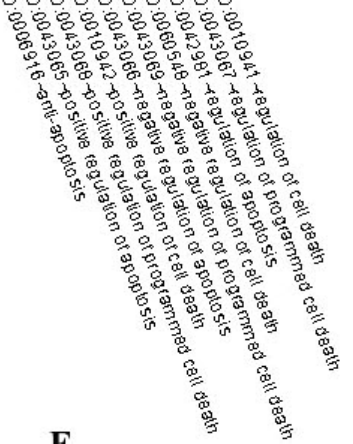

$\mathbf{F}$

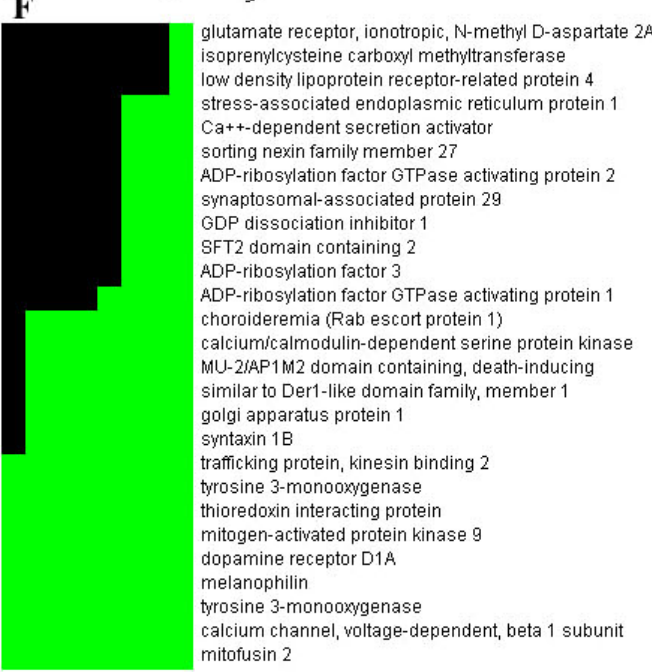

88888888

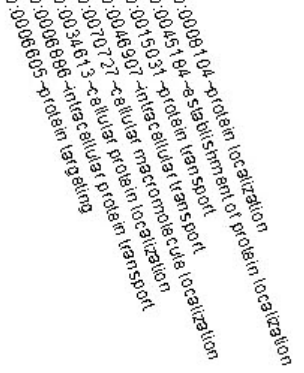

Figure 4. DAVID 2D view analysis of biologically significant FACs containing target genes of 20 months old VS 10 months old up-regulated miRNAs (>1.5 fold). (A) Synaptic function. (B) Cell fraction. (C) Regulation of secretion. (D) Apoptosis. (E) Response to hormone stimulus. (F) Protein localization. Green and black shading indicates positive and unconfirmed correlation of annotated gene and functional GO terms, respectively. 
of secretion, regulation of transport and regulation of position (Figure 4(C)). Among them, dopamine receptor D1A as an important part of the dopamine system, together with the catecholamine neurotransmitter dopamine, participate in motor function, learning and memory function, brain aging, and many other physiological activities. GluRs play vital role in learning, memory, neuronal plasticity, brain development and aging, etc., when bound with the important neurotransmitter glutamate.

\subsection{Apoptosis}

FAC analysis identified apoptosis as an important biological process during hippocampus ageing (Figure 1). DAVID heat map analyses identified B-cell translocation gene 2 (Bcl2), transforming growth factor (TGF), transforming growth factor- $\beta$ receptor 1 (TGF- $\beta$ R1), presenlin 1 , SMAD family member 3 (SMAD3), BDNF, caspase 3, B-cell translocation gene 2 etc. that functionally clustered into common GO terms related to apoptosis and other associated processes such as regulation of apoptosis, anti-apoptosis, regulation of cell death, positive and negative regulation of programmed cell death (Figure 4(D)).

Apoptosis suits itself into antagonistic pleiotropy theory and as it has deleterious effects in aging neurons while it is needed during development where it removes neurons that don't integrate into the growing neuronal network [26]. However, after the development of the neuronal network into the mature nervous system, the fight of neurons for survival and healthy existence with plasticity becomes anti-apoptotic [27]. In the mitochondrial pathway of apoptosis, intracellular signals result in releasing cytochrome $\mathrm{c}$ from mitochondria, which binds to the adaptor protein APAF-1 (apoptotic protease-activating factor-1), which further leads to the activation of Caspase 9 and subsequently Caspase 3 [28].

$\mathrm{Bcl} 2$ as a pro-survival gene is a member of the anti-apoptotic group, which binds to and inhibits the action of multi-domain pro-apoptotic proteins like Bax [29]. Moreover, the up-regulation of Bcl2 expression leads to down-regulation of Bax, and interferes with the release of cytochrome $\mathrm{c}$ from the mitochondrial intermembrane space into the cytosol, where it associates with Caspase 9 and Apaf1 to form the apoptosome complex, thus effecting apoptosis [30].

Therefore the regulation of miRNAs that target key genes such as Bcl2 expression may be interpreted as potential therapeutic candidates for preventing apoptotic death in neuronal cells, thus contributing to cognitive robustness.

\subsection{Response to Hormone Stimulus and Protein Localization}

DAVID FAC analysis indicated that response to hormone stimulus and protein localization is active in both adult and aged hippocampus (Figure 1, Figure 2). FAC analysis identified responses to hormone stimulus, endogenous stimulus, estrogen stimulus, estradiol stimulus, organic substance and alkaloid as significant biological processes during hippocampus ageing, dopamine receptor D1A, ras homolog gene family, insulin-like growth factor 2 , TGF- $\beta$ R1, regulator of 
calcineurin 1 , tachykinin receptor 1 , adenylate cyclase 6 , insulin induced gene 1 etc. were identified in the DAVID heat map (Figure 4(E)). DAVID heat map analysis identified 27 target genes of up-regulated miRNAs (Figure 4(F)) and 29 target genes of down-regulated miRNAs (not shown) involved in protein localization. These include dopamine receptor D1A, NMDA-R, mitogen-activated protein kinase 9 (MAPK9) etc.

Several in vitro studies demonstrate potent neurotrophic actions of IGF-1 and IGF-2 in both neurons and glia including stimulation of DNA and RNA synthesis, induction of neurite outgrowth, regulation of neurotransmitter release, synaptogenesis and protection against neurotoxic insults [31] [32]. During hippocampus ageing, organizations secretion, such as hormone will be changed, and miRNAs expression will be changed too, they would involve in tissue autocrine and response to secretion through the regulation of their targets.

DAVID heat map analyses also identified a cluster of predicted target genes found to be involved in neuron projection, synaptic transmission and regulation of kinase activity (not shown). Neuron projection and synaptic transmission are very important physiological activities in CNS; therefore these functions also show significant changes associated with ageing, of course, the related important cytokines will be regulated by miRNAs.

\subsection{Results of KEGG Pathway Analysis}

The important GO terms of FAC analysis were chosen for KEGG pathway analysis. Among them, neurotrophin signaling pathway, which widely exists in brain tissue, plays an important physiological role in all kinds of neural cells growth, differentiation, maintenance and regeneration (Figure 5(A))

(http://www.genome.jp/kegg-bin/show_pathway?mmu04722+12064). Long term potentiation (LTP) related signal pathways, widely existing in brain hippocampus, cortex, limbic system, cerebellum, midbrain, thalamus and other brain regions, are closely related to synaptic plasticity, learning and memory process (Figure 5(B)) (http://www.genome.jp/kegg-bin/show_pathway?mmu04720+14811). Cell apoptosis signaling pathway, which is closely related to the hippocampal growth and aging (Figure 5(C))

(http://www.genome.jp/kegg-bin/show_pathway?mmu04210+12043); TGF- $\beta$ signaling pathway, which can regulate multiple cellular functions, including cell growth, adhesion, cell transfer, differentiation and apoptosis, and plays an important role in regulating immune inflammation, wound healing, immune steady and tolerance (Figure 5(D))

(http://www.genome.jp/kegg-bin/show_pathway?mmu04350+21808). Dopamine synaptic pathway, which participates in the control of the movement in CNS, plays an important role in regulating brain development, ageing, learning and memory function (Figure $5(\mathrm{E})$ )

(http://www.genome.jp/kegg-bin/show_pathway?mmu04728+13488). In the KEGG pathway maps, red colour was used to mark the predicted target genes of 


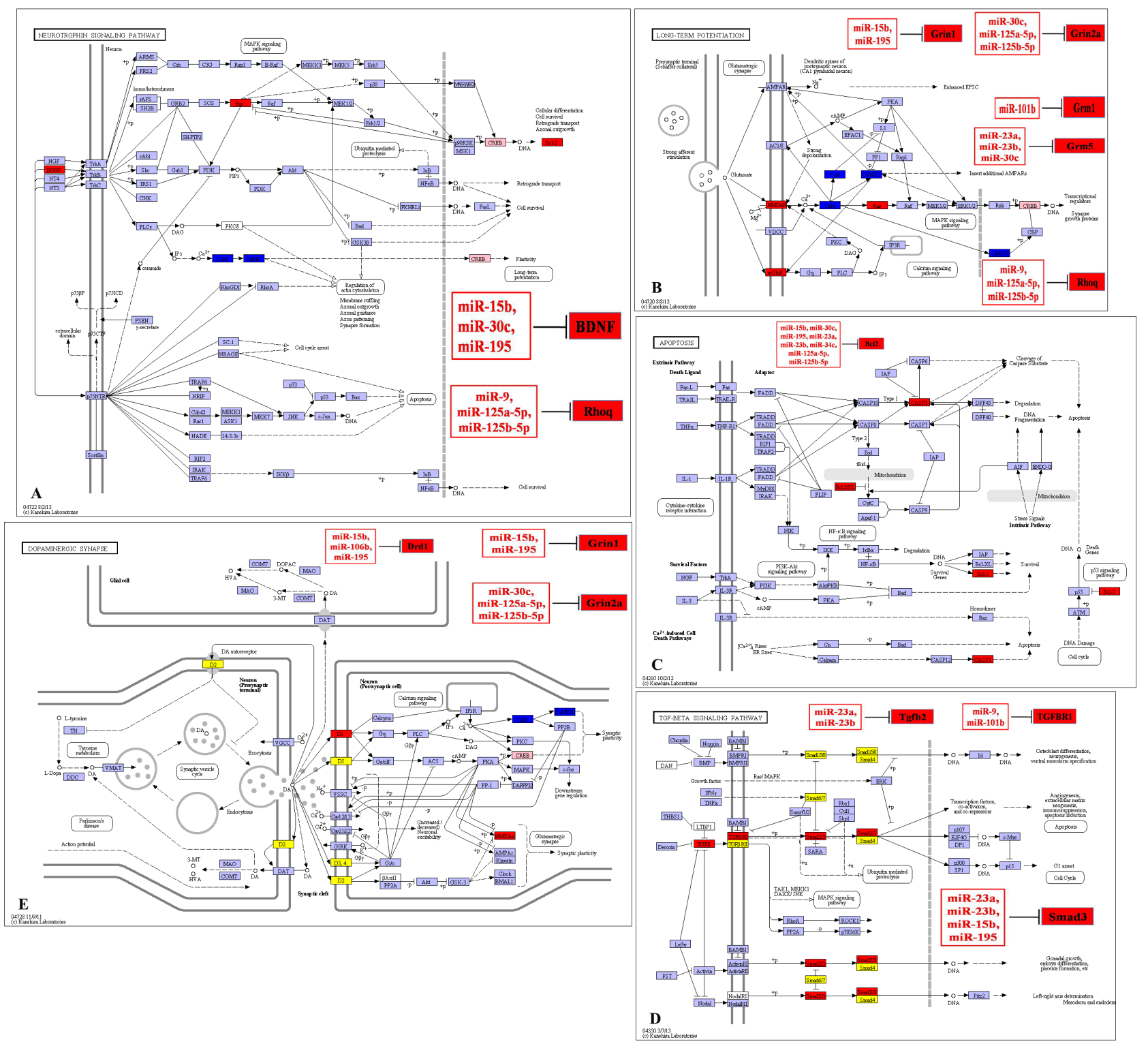

Figure 5. KEGG pathway map analyses of significant GO terms correlated with target genes of differentially expressed miRNAs. (A) Neurotrophin signal pathway. (B) LTP related signal pathways. (C) Apoptosis. (D) TGF- $\beta$ signal pathway. (E) Dopamine synaptic pathway.

miRNAs and differentially expressed miRNAs (Figure 5, Table 3). If further studies confirm these differentially expressed miRNAs (Table 3) could combine with mRNA 3'UTR sequence of predicted target genes and inhibited the expression of target genes, that suggests these miRNAs are involved in regulation of the above mentioned important signaling pathways by inhibiting the expression of the important cytokines, and attend in regulated hippocampal growth, ageing and maintenance, and then regulate behavior, emotion, learning and memory function.

\section{Discussion}

The hippocampus is an essential part of the archeo-cortex. In mammals, it is 
Table 3. List of miRNAs from Mirbase, Miranda and Mirdb with predicted target mRNAs KEGG ID, description and KEGG pathway.

\begin{tabular}{|c|c|c|c|}
\hline $\begin{array}{l}\text { KEGG } \\
\text { ID }\end{array}$ & Description & KEGG Pathway & miRNAs \\
\hline K04355 & dnf, brain derived neurophic factor & Neurotrophin signaling pathway & miR-15b, miR-195, miR-30c; \\
\hline K02161 & Bcl2, B-cell CLL/lymphoma 2 & Apoptosis & $\begin{array}{l}\text { miR-195, miR-23b, miR-23a, miR-34c, miR-15b, } \\
\text { miR-125b-5p, miR-125a-5p; }\end{array}$ \\
\hline K05209 & $\begin{array}{c}\text { Grin2a, glutamate receptor, } \mathrm{N} \text {-methyl } \\
\text { D-aspartate } 2 \mathrm{~A}\end{array}$ & Long term potentiation & miR-125a-5p, miR-125b-5p, miR-30c; \\
\hline K05208 & Grin1,glutamate receptor & Long term potentiation & miR-15b, miR-195; \\
\hline K04144 & Drd1, dopamine receptor D1A & Dopaminergic synapse & miR-15b, miR-106b, miR-195; \\
\hline K07828 & Rhoq, ras homolog family member Q & Long term potentiation & miR-125a-5p, miR-125b-5p, miR-9; \\
\hline K04500 & Smad3, SMAD family member 3 & TGF-beta signaling pathway & miR-23a, miR-15b, miR-23b, miR-9, miR-195, miR-101b; \\
\hline K13376 & Tgfb2, transforming growth factor, beta 2 & TGF-beta signaling pathway & $\mathrm{miR}-23 \mathrm{a}, \mathrm{miR}-23 \mathrm{~b}$ \\
\hline K04674 & TGFBR1, transforming growth factor & TGF-beta signaling pathway & miR-101b, miR-9, miR-128; \\
\hline K04603 & Grm1, glutamate receptor, metabotropic 1 & Long term potentiation & $\operatorname{miR}-101 b$ \\
\hline K04604 & Grm5, glutamate receptor, metabotropic 5 & Long term potentiation & miR-23a, miR-23b, miR-30c. \\
\hline
\end{tabular}

three-layered structure located on the medial surface of the temporal lobe in the back of each cerebral hemisphere. The name hippocampus is derived from Greek and means sea horse, which it resembles in shape [16]. The hippocampus is an important part of the limbic system, which plays vital role in the learning/memory, spatial navigation, emotional behavior and regulation of the neuroendocrine stress axis processes [16] [33].

In order to have comprehensive understanding of miRNAs expression differences and regulation functions in hippocampal ageing, 10 months old and 20 months old C57 mice were chosen, and two sets and a total of six hippocampal samples were sent to miRNAs microarray detection. 20 months old vs. 10 months old 1.5 fold change up and down regulated miRNAs (intersection of three sets of chip results) were chosen for further study (Table 1, Table 2). Through bioinformatic analysis, the predicted target genes of differentially expressed miRNAs are distributed in neurotrophic factor signal pathway, LTP related signal pathways, cell apoptosis signal pathway, transforming growth factor signal pathway and dopamine synaptic pathway, and many of these target genes are the significant cytokines of these signal pathways.

Neurotrophic factors, especially the BDNF can regulate the development, ageing and function of the CNS. BDNF has been implicated in activity dependent synaptic plasticity and network remodeling [34]. Moreover, it is able to regulate the extent of adult hippocampal neurogenesis [35], presumably via its specific TrkB receptors [36]. Proliferating neural progenitor cells in the dentate gyrus have been demonstrated to express TrkB receptors [37], suggesting a direct influence of BDNF on neurogenesis. It is well known that BDNF binding to 
TrkB receptors evokes several intracellular signaling pathways, including MAP/ ERK pathway and the activation of CREB [38]. BDNF is known to regulate dendritic development. In particular, it has been shown to induce primary dendrite formation in developing neurons via PI3-K and MAPK pathway activation [39]. Through bioinformation analysis, here from 10 months old to 20 months old ageing process, miR-15b, miR-195, miR-30c may bind with BDNF mRNA to participate in the regulation of neurotrophin signal pathway (Table 3, Figure 5(A)).

The hippocampus is a key brain area involved in learning and memory. Activitydependent forms of hippocampal synaptic plasticity, such as long-term potentiation (LTP) and long-term depression (LTD) are key cellular events underlying learning, memory and habituation [40]. Brain aging is generally accompanied by cognitive deficits, in particular by learning and memory impairments [41]. Age-related deficits in learning and memory could occur in parallel with the impairment of functional plasticity at central synapses. Experimental data confirm this assumption, because the expression of synaptic plasticity, including LTP and LTD of synaptic transmission, is altered in the brain of memory deficient aged animals [42] [43]. Through bioinformation analysis, here from 10 months old to 20 months old ageing process, miR-15b, miR-195 may bind with Grin1 mRNA; miR-30c, miR-125a-5p, miR-125b-5p may bind with Grin2a mRNA; miR-125a-5p, miR-125b-5p, miR-9 may bind with Rhoq mRNA; miR-101b may bind with Grm1 mRNA; and miR-23a, miR-23b, miR-30c may bind with Grm5 mRNA, and these miRNAs may be involved in the regulation of LTP related signal pathways (Table 3, Figure 5(B)).

It is well known that age-associated neurodegeneration is associated with increased neuronal apoptosis, as one mechanism contributing to cognitive dysfunctions [44] [45]. Caspase 3 and Bax up-regulation has been observed in patient samples and in classic animal neurodegenerative models such as stroke, $\mathrm{AD}, \mathrm{HD}$ (Huntington's disease) and PD [46]. The Bcl-2 protein resides in the outer mitochondrial wall, and acts as an anti-apoptotic factor by controlling mitochondrial permeability, thus regulating apoptosis [47]. Previous evidence of miRNA impact on cell survival mechanisms has been shown with miR-15, miR-16, miR-34a and miR-181a- ${ }^{*}$ targeting Bcl-2 mRNA and inducing apoptosis [48] [49]. Here from 10 months old to 20 months old ageing process, miR-15b, miR-195, miR-30c, miR-23a, miR-23b, miR-125a-5p, miR-125b-5p, miR-34c may bind with $\mathrm{Bcl} 2 \mathrm{mRNA}$ to participate in the regulation of apoptosis signal pathway (Table 3, Figure $5(\mathrm{C})$ ).

TGF- $\beta$ signal pathway, which can regulate multiple cellular functions, including cell growth, adhesion, cell transfer, differentiation and apoptosis, and play an important role in regulating immune inflammation, wound healing, immune steady and tolerance [50]. TGF- $\beta$ signal pathway was precision regulated in different levels, and dysfunction is closely related to the occurrence of a variety of diseases. Smad3 protein was the key molecule in TGF- $\beta$ signal transduction, it play an important role in maintaining the normal function of cells [51]. Through 
bioinformation analysis, here from 10 months old to 20 months old ageing process, miR-23a, miR-23b may bind with TGF- $\beta$ IImRNA; miR-9, miR-101b, miR-128 may bind with TGF- $\beta$ ImRNA; miR-9, miR-23a, miR-23b, miR-101b, miR-15b, miR-195 may bind with SMAD3 mRNA; and these miRNAs may be involved in the regulation of TGF- $\beta$ signal pathway (Table 3, Figure 5(D)).

Dopamine is the mainly catecholamine neurotransmitter in mammalian brain, It controls the motion, cognition, emotion, positive reinforcement, feeding and endocrine regulation and many other functions [52]. Dopamine receptors can be divided into D1 and D2 receptors. They are both expressed in hippocampus. Dopamine play an important role for motion control, and dopamine levels were positively correlated with cognitive function, and negatively correlated with age [53]. Through bioinformation analysis, here from 10 months old to 20 months old ageing process, miR-15b, miR-195 may bind with D1 mRNA to participate in the regulation of dopamine synaptic pathway (Table 3, Figure $5(\mathrm{E})$ ).

Increased age is a major risk factor for neurodegenerative disease incidence, post-ischemic mortality, and severe and long-term disability. Here from 10 months old to 20 months old ageing process, significantly differentially expressed miRNAs may bind with mRNA of their predicted target genes to participate in the regulation of neurotrophic factor signal pathway, LTP related signal pathways, apoptosis pathway, transforming growth factor signal pathway, dopamine synaptic pathway, and then regulate the hippocampal growth, maintenance, aging, degradation, learning and memory functions. This study lays a solid foundation for further studies to clarify the important regulation function of miRNAs in brain tissue, and will provide new diagnostic and therapeutic targets for effective prevention and treatment of neurodegenerative diseases.

\section{Acknowledgements}

This study was funded by The National Natural Science Foundation of China (81401095, Yang Liu); this study was funded by Hubei Province health and family planning scientific research project of China (WJ2015Q045, Yang Liu); this study was supported by The Yangtze Youth Fund (2015cqr24, Yang Liu).

\section{References}

[1] Lander, E.S. and Schork, N.J. (1994) Genetic Dissection of Complex Traits. Science, 265, 2037-2048. https://doi.org/10.1126/science.8091226

[2] Chihara, T., Kitabayashi, A., Morimoto, M., Takeuchi, K., Masuyama, K., Tonoki, A., et al. (2014) Caspase Inhibition in Select Olfactory Neurons Restores Innate Attraction Behavior in Aged Drosophila. PLOS Genetics, 10, e1004437. https://doi.org/10.1371/journal.pgen.1004437

[3] Lee, Y., Kim, M., Han, J., Yeom, K.H., Lee, S., Baek, S.H., et al. (2004) MicroRNA Genes Are Transcribed by RNA Polymerase II. EMBO Journal, 23, 4051-4060. https://doi.org/10.1038/sj.emboj.7600385

[4] Hausser, J., Landthaler, M., Jaskiewicz, L., Gaidatzis, D. and Zavolan, M. (2009) Relative Contribution of Sequence and Structure Features to the mRNA Binding of Argonaute/EIF2C-miRNA Complexes and the Degradation of miRNA Targets. 
Genome Research, 19, 2009-2020. https://doi.org/10.1101/gr.091181.109

[5] Guo, H., Ingolia, N.T., Weissman, J.S. and Bartel, D.P. (2010) Mammalian micrornas Predominantly Act to Decrease Target mRNA Levels. Nature, 466, 835-840. https://doi.org/10.1038/nature09267

[6] Lee, R.C., Feinbaum, R.L. and Ambros, V. (1993) The C. elegans Heterochronic Gene Lin-4 Encodes Small RNAs with Antisense Complementarity to Lin-14. Cell, 75, 843-854. https://doi.org/10.1016/0092-8674(93)90529-Y

[7] Edbauer, D., Neilson, J.R., Foster, K.A., Wang, C.F., Seeburg, D.P., Batterton, M.N., et al. (2010) Regulation of Synaptic Structure and Function by FMRP-Associated microRNAs miR-125b and miR-132. Neuron, 65, 373-384. https://doi.org/10.1016/j.neuron.2010.01.005

[8] Chandrasekar, V. and Dreyer, J.L. (2011) Regulation of MiR-124, Let-7d, and MiR-181a in the Accumbens Affects the Expression, Extinction, and Reinstatement of Cocaine-Induced Conditioned Place Preference. Neuropsychopharmacology, 36, 1149-1164. https://doi.org/10.1038/npp.2010.250

[9] Yang, Z., Jun, H., Choi, C.I., Yoo, K.H., Cho, C.H., Hussaini, S.M.Q., et al. (2017) Age-Related Decline in BubR1 Impairs Adult Hippocampal Neurogenesis. Aging Cell, 16, 598-601. https://doi.org/10.1111/acel.12594

[10] Maes, O.C., Chertkow, H.M., Wang, E. and Schipper, H.M. (2009) MicroRNA: Implications for Alzheimer Disease and Other Human CNS Disorders. Current Genomics, 10, 154-168. https://doi.org/10.2174/138920209788185252

[11] Xu, S., Witmer, P.D., Lumayag, S., Kovacs, B. and Valle, D. (2007) MicroRNA (miRNA) Transcriptome of Mouse Retina and Identification of a Sensory Organ-Specific miRNA Cluster. The Journal of Biological Chemistry, 282, 25053-25066. https://doi.org/10.1074/jbc.M700501200

[12] Garzon, R., Pichiorri, F., Palumbo, T., Visentini, M., Aqeilan, R., Cimmino, A., et al. (2007) MicroRNA Gene Expression during Retinoic Acid-Induced Differentiation of Human Acute Promyelocytic Leukemia. Oncogene, 26, 4148-4157. https://doi.org/10.1038/sj.onc.1210186

[13] Caravia, X.M., Roiz-Valle, D., Moran-Alvarez, A. and Lopez-Otin, C. (2017) Functional Relevance of miRNAS IN Premature Ageing. Mechanisms of Ageing and Development. (In Press) https://doi.org/10.1016/j.mad.2017.05.003

[14] Krol, J., Loedige, I. and Filipowicz, W. (2010) The Widespread Regulation of Microrna Biogenesis, Function and Decay. Nature Reviews Genetics, 11, 597-610. https://doi.org/10.1038/nrg2843

[15] Meza-Sosa, K.F., Valle-Garcia, D., Pedraza-Alva, G. and Perez-Martinez, L. (2012) Role of microRNAs in Central Nervous System Development and Pathology. Journal of Neuroscience Research, 90, 1-12. https://doi.org/10.1002/jnr.22701

[16] Sendrowski, K. and Sobaniec, W. (2013) Hippocampus, Hippocampal Sclerosis and Epilepsy. Pharmacological Reports, 65, 555-565. https://doi.org/10.1016/S1734-1140(13)71033-8

[17] Sun, L.Y., Evans, M.S., Hsieh, J., Panici, J. and Bartke, A. (2005) Increased Neurogenesis in Dentate Gyrus of Long-Lived Ames Dwarf Mice. Endocrinology, 146, 1138-1144. https://doi.org/10.1210/en.2004-1115

[18] Eisen, M.B., Spellman, P.T., Brown, P.O. and Botstein, D. (1998) Cluster Analysis and Display of Genome-Wide Expression Patterns. Proceedings of the National Academy of Sciences, 95, 14863-14868. https://doi.org/10.1073/pnas.95.25.14863

[19] Raychaudhuri, S., Stuart, J.M. and Altman, R.B. (2000) Principal Components Analysis to Summarize Microarray Experiments: Application to Sporulation Time Series. Pacific Symposium on Biocomputing, 455-466.

[20] Huang da, W., Sherman, B.T. and Lempicki, R.A. (2009) Systematic and Integrative Analysis of Large Gene Lists using DAVID Bioinformatics Resources. Nature Pro- 
tocols, 4, 44-57. https://doi.org/10.1038/nprot.2008.211

[21] Izquierdo, I. (1991) Role of NMDA Receptors in Memory. Trends in Pharmacological Sciences, 12, 128-129. https://doi.org/10.1016/0165-6147(91)90527-Y

[22] Turpin, F.R., Potier, B., Dulong, J.R., Sinet, P.M., Alliot, J., Oliet, S.H., et al. (2011) Reduced Serine Racemase Expression Contributes to Age-Related Deficits in Hippocampal Cognitive Function. Neurobiology of Aging, 32, 1495-1504. https://doi.org/10.1016/j.neurobiolaging.2009.09.001

[23] Mattson, M.P. (2008) Glutamate and Neurotrophic Factors in Neuronal Plasticity and Disease. Annals of the New York Academy of Sciences, 1144, 97-112. https://doi.org/10.1196/annals.1418.005

[24] Lee, E. and Son, H. (2009) Adult Hippocampal Neurogenesis and Related Neurotrophic Factors. BMB Reports, 42, 239-244. https://doi.org/10.5483/BMBRep.2009.42.5.239

[25] Cohen-Cory, S., Kidane, A.H., Shirkey, N.J. and Marshak, S. (2010) Brain-Derived Neurotrophic Factor and the Development of Structural Neuronal Connectivity. Developmental Neurobiology, 70, 271-288. https://doi.org/10.1002/dneu.20774

[26] Ranger, A.M., Malynn, B.A. and Korsmeyer, S.J. (2001) Mouse Models of Cell Death. Nature Genetics, 28, 113-118. https://doi.org/10.1038/88815

[27] Germain, M. and Slack, R.S. (2010) Dining in with BCL-2: New Guests at the Autophagy Table. Clinical Science, 118, 173-181. https://doi.org/10.1042/CS20090310

[28] Li, J. and Yuan, J. (2008) Caspases in Apoptosis and Beyond. Oncogene, 27, 6194-6206. https://doi.org/10.1038/onc.2008.297

[29] Cory, S. and Adams, J.M. (2002) The Bcl2 Family: Regulators of the Cellular Life-or-Death Switch. Nature Reviews Cancer, 2, 647-656. https://doi.org/10.1038/nrc883

[30] Zou, H., Li, Y., Liu, X. and Wang, X. (1999) An APAF-1. Cytochrome c Multimeric Complex Is a Functional Apoptosome That Activates Procaspase-9. The Journal of Biological Chemistry, 274, 11549-11556. https://doi.org/10.1074/jbc.274.17.11549

[31] Dore, S., Kar, S. and Quirion, R. (1997) Insulin-Like Growth Factor I Protects and Rescues Hippocampal Neurons against Beta-Amyloid and Human Amylin-Induced Toxicity. Proceedings of the National Academy of Sciences, 94, 4772-4777. https://doi.org/10.1073/pnas.94.9.4772

[32] Sun, L.Y., Al-Regaiey, K., Masternak, M.M., Wang, J. and Bartke, A. (2005) Local Expression of GH and IGF-1 in the Hippocampus of GH-Deficient Long-Lived Mice. Neurobiology of Aging, 26, 929-937. https://doi.org/10.1016/j.neurobiolaging.2004.07.010

[33] Tanti, A. and Belzung, C. (2013) Neurogenesis along the Septo-Temporal axis of the Hippocampus: Are Depression and the Action of Antidepressants Region-Specific? Neuroscience. https://doi.org/10.1016/j.neuroscience.2013.08.017

[34] Kuczewski, N., Porcher, C., Lessmann, V., Medina, I. and Gaiarsa, J.L. (2009) Activity-Dependent Dendritic Release of BDNF and Biological Consequences. Molecular Neurobiology, 39, 37-49. https://doi.org/10.1007/s12035-009-8050-7

[35] Rossi, C., Angelucci, A., Costantin, L., Braschi, C., Mazzantini, M., Babbini, F., et al. (2006) Brain-Derived Neurotrophic Factor (BDNF) Is Required for the Enhancement of Hippocampal Neurogenesis following Environmental Enrichment. European Journal of Neuroscience, 24, 1850-1856. https://doi.org/10.1111/j.1460-9568.2006.05059.x

[36] Donovan, M.H., Yamaguchi, M. and Eisch, A.J. (2008) Dynamic Expression of TrkB Receptor Protein on Proliferating and Maturing Cells in the Adult Mouse Dentate Gyrus. Hippocampus, 18, 435-439. https://doi.org/10.1002/hipo.20410

[37] Li, Y., Luikart, B.W., Birnbaum, S., Chen, J., Kwon, C.H., Kernie, S.G., et al. (2008) 
TrkB Regulates Hippocampal Neurogenesis and Governs Sensitivity to Antidepressive Treatment. Neuron, 59, 399-412. https://doi.org/10.1016/j.neuron.2008.06.023

[38] Numakawa, T., Suzuki, S., Kumamaru, E., Adachi, N., Richards, M. and Kunugi, H. (2010) BDNF Function and Intracellular Signaling in Neurons. Histology and Histopathology, 25, 237-258.

[39] Dijkhuizen, P.A. and Ghosh, A. (2005) BDNF Regulates Primary Dendrite Formation in Cortical Neurons via the PI3-Kinase and MAP Kinase Signaling Pathways. Journal of Neurobiology, 62, 278-288. https://doi.org/10.1002/neu.20100

[40] Bliss, T.V. and Collingridge, G.L. (1993) A Synaptic Model of Memory: Long-Term Potentiation in the Hippocampus. Nature, 361, 31-39. https://doi.org/10.1038/361031a0

[41] Billard, J.M. (2006) Ageing, Hippocampal Synaptic Activity and Magnesium. Magnesium Research, 19, 199-215.

[42] Potier, B., Turpin, F.R., Sinet, P.M., Rouaud, E., Mothet, J.P., Videau, C., et al. (2010) Contribution of the d-Serine-Dependent Pathway to the Cellular Mechanisms Underlying Cognitive Aging. Frontiers in Aging Neuroscience, 2, 1. https://doi.org/10.3389/neuro.24.001.2010

[43] Edelmann, E., Cepeda-Prado, E. and Lessmann, V. (2017) Coexistence of Multiple Types of Synaptic Plasticity in Individual Hippocampal CA1 Pyramidal Neurons. Frontiers in Synaptic Neuroscience, 9, 7. https://doi.org/10.3389/fnsyn.2017.00007

[44] Zhu, X., Raina, A.K., Perry, G. and Smith, M.A. (2006) Apoptosis in Alzheimer Disease: A Mathematical Improbability. Current Alzheimer Research, 3, 393-396. https://doi.org/10.2174/156720506778249470

[45] Moran, C., Beare, R., Phan, T., Starkstein, S., Bruce, D., Romina, M., et al. (2017) Neuroimaging and Its Relevance to Understanding Pathways Linking Diabetes and Cognitive Dysfunction. Journal of Alzheimer's Disease, 59, 405-419.

[46] Mattson, M.P. (2006) Neuronal Life-and-Death Signaling, Apoptosis, and Neurodegenerative Disorders. Antioxidants \& Redox Signaling, 8, 1997-2006. https://doi.org/10.1089/ars.2006.8.1997

[47] Osford, S.M., Dallman, C.L., Johnson, P.W., Ganesan, A. and Packham, G. (2004) Current Strategies to Target the Anti-Apoptotic Bcl-2 Protein in Cancer Cells. Current Medicinal Chemistry, 11, 1031-1039. https://doi.org/10.2174/0929867043455486

[48] Khanna, A., Muthusamy, S., Liang, R., Sarojini, H. and Wang, E. (2011) Gain of Survival Signaling by Down-Regulation of Three Key miRNAs in Brain of Calorie-Restricted Mice. Aging, 3, 223-236. https://doi.org/10.18632/aging.100276

[49] Cimmino, A., Calin, G.A., Fabbri, M., Iorio, M.V., Ferracin, M., Shimizu, M., et al. (2005) miR-15 and miR-16 Induce Apoptosis by Targeting BCL2. Proceedings of the National Academy of Sciences, 102, 13944-13949.

https://doi.org/10.1073/pnas.0506654102

[50] Schmierer, B. and Hill, C.S. (2007) TGFbeta-SMAD Signal Transduction: Molecular Specificity and Functional Flexibility. Nature Reviews Molecular Cell Biology, 8, 970-982. https://doi.org/10.1073/pnas.0506654102

[51] Lin, X., Duan, X., Liang, Y.Y., Su, Y., Wrighton, K.H., Long, J., et al. (2006) PPM1A Functions as a Smad Phosphatase to Terminate TGFbeta Signaling. Cell, 125, 915-928. https://doi.org/10.1016/j.cell.2006.03.044

[52] Jackson, D.M. and Westlind-Danielsson, A. (1994) Dopamine Receptors: Molecular Biology, Biochemistry and Behavioural Aspects. Pharmacology \& Therapeutics, 64, 291-370. https://doi.org/10.1016/0163-7258(94)90041-8

[53] De Keyser, J., De Backer, J.P., Vauquelin, G. and Ebinger, G. (1990) The Effect of Aging on the D1 Dopamine Receptors in Human Frontal Cortex. Brain Research, 528, 308-310. https://doi.org/10.1016/0006-8993(90)91672-4 